\title{
Contact patterns and their implied basic reproductive numbers: an illustration for varicella-zoster virus
}

\author{
T. VAN EFFELTERRE ${ }^{1 *}$, Z. SHKEDY ${ }^{1}$, M. AERTS ${ }^{1}$, G. MOLENBERGHS ${ }^{1}$, \\ P. VAN DAMME ${ }^{2}$ AND P. BEUTELS ${ }^{2,3}$ \\ ${ }^{1}$ Hasselt University, Center for Statistics, Biostatistics, Diepenbeek, Belgium \\ ${ }^{2}$ University of Antwerp, Epidemiology and Social Medicine, Center for Evaluation of Vaccination, Antwerp, \\ Belgium \\ ${ }^{3}$ National Centre for Immunization Research and Surveillance, University of Sydney, Australia
}

(Accepted 25 February 2008; first published online 9 May 2008)

\section{SUMMARY}

The WAIFW matrix (Who Acquires Infection From Whom) is a central parameter in modelling the spread of infectious diseases. The calculation of the basic reproductive number $\left(R_{0}\right)$ depends on the assumptions made about the transmission within and between age groups through the structure of the WAIFW matrix and different structures might lead to different estimates for $R_{0}$ and hence different estimates for the minimal immunization coverage needed for the elimination of the infection in the population. In this paper, we estimate $R_{0}$ for varicella in Belgium. The force of infection is estimated from seroprevalence data using fractional polynomials and we show how the estimate of $R_{0}$ is heavily influenced by the structure of the WAIFW matrix.

\section{INTRODUCTION}

An essential assumption in modelling the spread of infectious diseases is that the force of infection, which is the probability for a susceptible to acquire the infection, varies over time as a function of the level of infectivity in the population [1]. For many infectious diseases, the force of infection is also known to depend on age. The equation describing the dependence of the force of infection on age and time is given by

$$
\lambda(a, t)=\int_{0}^{L} \beta\left(a, a^{\prime}\right) I\left(a^{\prime}, t\right) \mathrm{d} a^{\prime} .
$$

The coefficients $\beta\left(a, a^{\prime}\right)$ are called the transmission coefficients and $I\left(a^{\prime}, t\right)$ is the number of infectious individuals at age $a^{\prime}$ and time $t$. These transmission coefficients combine epidemiological, environmental

\footnotetext{
* Author for correspondence: Dr T. Van Effelterre, Hasselt University, Center for Statistics, Biostatistics, Agoralaan 1, B3590 Diepenbeek, Belgium.

(Email: tvaneff@yahoo.com)
}

and social factors affecting the transmission rate between an infective of age $a^{\prime}$ and a susceptible of age $a$ $[1,2]$. For the discrete case with a population divided into a finite number, say $n$, of age groups, Anderson \& May [3] introduced the WAIFW (Who Acquires Infection From Whom) matrix in which the $i j$ th entry of the matrix, $\beta_{i j}$, is the transmission coefficient from an infective in age group $j$ to a susceptible in age group $i$. Let $\bar{I}_{i}$ be the total number of infectious individuals in the $i$ th age group at time $t, i=1, \ldots, n$, then the age- and time-dependent force of infection can be approximated by the matrix product

$\lambda=W \bar{I}$.

Here, $\bar{I}=\left(\bar{I}_{1}, \ldots, \bar{I}_{n}\right)$ is the vector in which the $i$ th element is the number of infectious individuals (prevalence of infectivity) in age group $i, \lambda=\left(\lambda_{1}, \ldots, \lambda_{n}\right)$ is the vector in which the $i$ th element is the force of infection specific to age group $i$ and $W$ is a known WAIFW matrix. The configuration of the WAIFW matrix represents a priori knowledge (or assumptions) 
about the mixing patterns in the population. Several configurations are discussed in the literature (see e.g. $[1,2,4-6])$. For example, for a model with five age groups the WAIFW matrix $W_{1}$ in equation (3) represents a mixing pattern for which individuals are mixing only with individuals from their own age group (assortative mixing [5]) with a specific age-dependent transmission coefficient while $W_{2}$ represents a mixing pattern similar to $W_{1}$, also accounting for an additional mixing of individuals with individuals of other age groups with a 'background' transmission coefficient:

$W_{1}=\left(\begin{array}{ccccc}\beta_{1} & 0 & 0 & 0 & 0 \\ 0 & \beta_{2} & 0 & 0 & 0 \\ 0 & 0 & \beta_{3} & 0 & 0 \\ 0 & 0 & 0 & \beta_{4} & 0 \\ 0 & 0 & 0 & 0 & \beta_{5}\end{array}\right), \quad W_{2}=\left(\begin{array}{ccccc}\beta_{1} & \beta_{5} & \beta_{5} & \beta_{5} & \beta_{5} \\ \beta_{5} & \beta_{2} & \beta_{5} & \beta_{5} & \beta_{5} \\ \beta_{5} & \beta_{5} & \beta_{3} & \beta_{5} & \beta_{5} \\ \beta_{5} & \beta_{5} & \beta_{5} & \beta_{4} & \beta_{5} \\ \beta_{5} & \beta_{5} & \beta_{5} & \beta_{5} & \beta_{5}\end{array}\right)$

Note that both matrices have five unknown parameters and both are symmetric. For each of these contact structures, the number of parameters is equal
The basic reproductive number $R_{0}$ can be computed as the dominant eigenvalue of a matrix for which the $i j$ th entry is the basic reproductive number $R_{0_{i j}}$, specific to the transmission from an infective in age group $j$ to a susceptible in age group $i$. More precisely, $R_{0_{i j}}=\beta_{i, j} D N_{i}$ where $D$ is the duration of infectiousness assumed independent of age and $N_{i}$ is the size of the population in age group $i$. Therefore, the estimator for $R_{0}$ depends on the configuration of the WAIFW matrix. Farrington et al. [4] showed that different configurations of the WAIFW matrix can lead to quite different estimates for $R_{\mathbf{0}}$. For example, Farrington

$$
W_{3}=\left(\begin{array}{lllll}
\beta_{1} & \beta_{1} & \beta_{4} & \beta_{4} & \beta_{5} \\
\beta_{1} & \beta_{2} & \beta_{4} & \beta_{4} & \beta_{5} \\
\beta_{4} & \beta_{4} & \beta_{3} & \beta_{4} & \beta_{5} \\
\beta_{4} & \beta_{4} & \beta_{4} & \beta_{4} & \beta_{5} \\
\beta_{5} & \beta_{5} & \beta_{5} & \beta_{5} & \beta_{5}
\end{array}\right), \quad W_{4}=\left(\begin{array}{llllll}
\beta_{1} & \beta_{1} & \beta_{3} & \beta_{4} & \beta_{5} \\
\beta_{1} & \beta_{2} & \beta_{3} & \beta_{4} & \beta_{5} \\
\beta_{3} & \beta_{3} & \beta_{3} & \beta_{4} & \beta_{5} \\
\beta_{4} & \beta_{4} & \beta_{4} & \beta_{4} & \beta_{5} \\
\beta_{5} & \beta_{5} & \beta_{5} & \beta_{5} & \beta_{5}
\end{array}\right) .
$$

to the number of age groups. This is a condition to have a solution [3]. Suppose that the population is divided into $n$ age groups and let $\hat{\lambda}_{=}\left(\hat{\lambda}_{1}, \ldots, \hat{\lambda}_{n}\right)$ be the estimated vector of age-specific force of infection in each age group. If the structure of the WAIFW matrix is known and consists of $n$ unknown parameters, the WAIFW matrix can be estimated using the equality

$$
\left(\begin{array}{l}
\hat{\lambda}_{1} \\
\cdot \\
\cdot \\
\hat{\lambda}_{n}
\end{array}\right)=\frac{N D}{L} W\left(\begin{array}{l}
\Psi_{1} \\
\cdot \\
\cdot \\
\Psi_{n}
\end{array}\right)
$$

with $N$ the total population size, $D$ the mean duration of infectiousness, $L$ the life-expectancy at birth, and

$$
\Psi_{j}=\mathrm{e}^{-\varphi_{j-1}}-\mathrm{e}^{-\varphi_{j}} \quad \text { and } \quad \varphi_{j}=\sum_{i=1}^{j} \hat{\lambda}_{i}\left(a_{i}-a_{i-1}\right) .
$$

Here, $a_{i}-a_{i-1}$ is the width of the $i$ th age group. Hence, as long as the WAIFW matrix has a known configuration with $n$ unknown parameters, the parameter vector $\beta=\left(\beta_{1}, \ldots, \beta_{n}\right)$ is identifiable. Note that we expect that $\beta_{i} \geqslant 0, i=1,2, \ldots, n$. et al. [4] estimated $R_{0}$ for mumps to be equal to $25 \cdot 5$, 8.0 and 3.3 for the configuration of $W_{2}, W_{3}$ and $W_{4}$, respectively:

Hence, the uncertainty related to the WAIFW matrix is coming from two different sources: (1) the uncertainty about the unknown transmission coefficients $\beta_{i}$ and (2) the uncertainty about the configuration of the WAIFW matrix. Furthermore, Wallinga et al. [6] showed that the basic reproductive number for measles ranges between $770 \cdot 38$ when assortative mixing pattern is assumed and 1.43 when infant mixing is assumed, i.e. infants are assumed to be the source of all infection [6].

In this paper, we present an investigation of the estimation of the basic reproductive number, $R_{0}$, and $p_{c}$, the minimal proportion of the population that needs to be vaccinated to eliminate the infection, for varicella in Belgium for which, currently, there is no vaccination programme. Following the approach of Greenhalgh \& Dietz [5] we show that, depending on our assumption about the contact patterns, $R_{0}$ ranges between 3.12 and 68.57 , and $p_{c}$ ranges between $67.9 \%$ and $98.5 \%$.

This paper is organized as follow. In the next section, we present six possible configurations for the WAIFW 
matrix for varicella and discuss the estimation of the age-dependent force of infection from serological data using fractional polynomials. In the 'Estimation of the transmission coefficients' section, the WAIFW matrices are estimated using the integrated force of infection in the relevant age groups. Parametric and non-parametric bootstrap is used to calculate confidence intervals for the transmission coefficients. The estimation of $R_{\mathbf{0}}$ and $p_{c}$ is discussed in the 'Estimation of $R_{0}$ from the WAIFW matrices' section.

\section{Estimation of $\boldsymbol{R}_{\mathbf{0}}$ and the WAIFW matrix for varicella based on serological data}

\section{Age-dependent transmission coefficients}

Several authors $[4,5,7]$ illustrated how the estimation of $R_{0}$ is influenced by the configuration of the WAIFW matrix. A specific configuration of the WAIFW matrix represents specific assumptions about age-dependent transmission coefficients in the population which in turn represents prior assumptions about the mixing patterns in the population.

We illustrate these concepts for varicella in Belgium. The population was divided into the following six age groups, taking into account the schooling system in Belgium, 6 months-1 year, 2-5 years, 6-11 years, 12-18 years, 19-30 years and 31-44 years. Several configurations were discussed by Anderson \& May [1] and Greenhalgh \& Dietz [5]. Assortative mixing assumes that all contacts occurs within the age groups [5]. The matrix $W_{V 1}$ has specific transmission coefficients within each age group, i.e. for the transmission among hosts belonging to the same age group on the diagonal, and of a common 'background' transmission coefficient between any two different age groups. This 'background' transmission coefficient is assumed equal to the transmission coefficients in the oldest age group $\left(\beta_{6}\right)$. Note that the transmission coefficient in the oldest age group and the 'background' transmission coefficient between different age groups are both expected to be smaller than the transmission coefficients in younger age groups. The second configuration, $W_{V 2}$, assumes that the main route of transmission for a directly transmitted viral infection like varicella is in kindergarten children or in the classroom. This is expressed by a unique coefficient $\beta_{2}$ for the (presumed high) transmission between infectious and susceptible hosts in the range $2-5$ years and two other specific coefficients $\left(\beta_{1}\right.$ and $\left.\beta_{3}\right)$ for transmission amongst other hosts aged $<12$ years. The third configuration of the WAIFW matrix, $W_{V 3}$ is a minor variation of the structure $W_{V 2}$ with a common transmission coefficient $\beta_{1}$ between hosts in the range 6 months-1 year and hosts aged $<12$ years. For the fourth configuration, $W_{V 4}$, the transmission coefficient depends only on the age group of the susceptible host. The susceptible hosts of a given age group are assumed to be as likely to acquire infection from infectious hosts of any age. Note that this structure is not symmetric. The fifth matrix structure, $W_{V 5}$, is a minor variation of the assortative structure $W_{V 1}$ in which there is a common transmission coefficient in the two older age groups and a distinct 'background' coefficient for the transmission between any other two different age groups. Finally, the sixth configuration, $W_{V 6}$, is the extreme case of assortative mixing for which there is only transmission assumed between hosts belonging to the same age group. Of these proposed configurations, the latter structure is most obviously unrealistic, it is nevertheless useful as it provides an upper bound to the basic reproductive number $R_{0}$ [5].

$$
\begin{gathered}
W_{V 1}=\left(\begin{array}{llllll}
\beta_{1} & \beta_{6} & \beta_{6} & \beta_{6} & \beta_{6} & \beta_{6} \\
\beta_{6} & \beta_{2} & \beta_{6} & \beta_{6} & \beta_{6} & \beta_{6} \\
\beta_{6} & \beta_{6} & \beta_{3} & \beta_{6} & \beta_{6} & \beta_{6} \\
\beta_{6} & \beta_{6} & \beta_{6} & \beta_{4} & \beta_{6} & \beta_{6} \\
\beta_{6} & \beta_{6} & \beta_{6} & \beta_{6} & \beta_{5} & \beta_{6} \\
\beta_{6} & \beta_{6} & \beta_{6} & \beta_{6} & \beta_{6} & \beta_{6}
\end{array}\right), \quad W_{V 2}=\left(\begin{array}{lllllll}
\beta_{1} & \beta_{1} & \beta_{3} & \beta_{4} & \beta_{5} & \beta_{6} \\
\beta_{1} & \beta_{2} & \beta_{3} & \beta_{4} & \beta_{5} & \beta_{6} \\
\beta_{3} & \beta_{3} & \beta_{3} & \beta_{4} & \beta_{5} & \beta_{6} \\
\beta_{4} & \beta_{4} & \beta_{4} & \beta_{4} & \beta_{5} & \beta_{6} \\
\beta_{5} & \beta_{5} & \beta_{5} & \beta_{5} & \beta_{5} & \beta_{6} \\
\beta_{6} & \beta_{6} & \beta_{6} & \beta_{6} & \beta_{6} & \beta_{6}
\end{array}\right), \\
W_{V 3}=\left(\begin{array}{llllll}
\beta_{1} & \beta_{1} & \beta_{1} & \beta_{4} & \beta_{5} & \beta_{6} \\
\beta_{1} & \beta_{2} & \beta_{3} & \beta_{4} & \beta_{5} & \beta_{6} \\
\beta_{1} & \beta_{3} & \beta_{3} & \beta_{4} & \beta_{5} & \beta_{6} \\
\beta_{4} & \beta_{4} & \beta_{4} & \beta_{4} & \beta_{5} & \beta_{6} \\
\beta_{5} & \beta_{5} & \beta_{5} & \beta_{5} & \beta_{5} & \beta_{6} \\
\beta_{6} & \beta_{6} & \beta_{6} & \beta_{6} & \beta_{6} & \beta_{6}
\end{array}\right), \quad W_{V 4}=\left(\begin{array}{lllllll}
\beta_{1} & \beta_{1} & \beta_{1} & \beta_{1} & \beta_{1} & \beta_{1} \\
\beta_{2} & \beta_{2} & \beta_{2} & \beta_{2} & \beta_{2} & \beta_{2} \\
\beta_{3} & \beta_{3} & \beta_{3} & \beta_{3} & \beta_{3} & \beta_{3} \\
\beta_{4} & \beta_{4} & \beta_{4} & \beta_{4} & \beta_{4} & \beta_{4} \\
\beta_{5} & \beta_{5} & \beta_{5} & \beta_{5} & \beta_{5} & \beta_{5} \\
\beta_{6} & \beta_{6} & \beta_{6} & \beta_{6} & \beta_{6} & \beta_{6}
\end{array}\right), \\
\end{gathered}
$$




$$
W_{V 5}=\left(\begin{array}{cccccc}
\beta_{1} & \beta_{6} & \beta_{6} & \beta_{6} & \beta_{6} & \beta_{6} \\
\beta_{6} & \beta_{2} & \beta_{6} & \beta_{6} & \beta_{6} & \beta_{6} \\
\beta_{6} & \beta_{6} & \beta_{3} & \beta_{6} & \beta_{6} & \beta_{6} \\
\beta_{6} & \beta_{6} & \beta_{6} & \beta_{4} & \beta_{6} & \beta_{6} \\
\beta_{6} & \beta_{6} & \beta_{6} & \beta_{6} & \beta_{5} & \beta_{6} \\
\beta_{6} & \beta_{6} & \beta_{6} & \beta_{6} & \beta_{6} & \beta_{5}
\end{array}\right), \quad W_{V 6}=\left(\begin{array}{cccccc}
\beta_{1} & 0 & 0 & 0 & 0 & 0 \\
0 & \beta_{2} & 0 & 0 & 0 & 0 \\
0 & 0 & \beta_{3} & 0 & 0 & 0 \\
0 & 0 & 0 & \beta_{4} & 0 & 0 \\
0 & 0 & 0 & 0 & \beta_{5} & 0 \\
0 & 0 & 0 & 0 & 0 & \beta_{6}
\end{array}\right) .
$$

\section{Estimation of the age-dependent force of infection}

\section{for varicella}

The estimation of the WAIFW matrix requires the estimation of the force of infection from prevaccination data. Following the methodology proposed by Anderson \& May [1] (see also [2, 7]) we assume that an age-specific serological profile can be estimated from pre-vaccination data. This can be done by modelling pre-vaccination seroprevalence data. The age-dependent force of infection can be derived from the estimated model for the prevalence of seropositive hosts by using non-parametric methods (discussed in $[8,9]$ ), or parametric methods (discussed in [4, 10-13]).

For varicella, we estimated the force of infection by using a seroprevalence dataset consisting of 1673 individuals aged between 1 and 44 years that was sampled in Antwerp (Belgium) between October 1999 and April 2000 and reported by Thiry et al. [14]. The sera were residual specimens submitted to medical laboratories for diagnostic purposes. Sera for the 1-11 years age group were collected from outpatients hospitals in Antwerp, sera for the 12-18 years age group were collected from volunteers in vaccine trials and sera for age groups $>16$ years were provided by a medical laboratory in Antwerp. The population was stratified by age in order to sample about 100 observations per age group. The force of infection can be estimated from this serological sample under the assumption that the disease is in a steady state.

For the analysis presented in this paper, fractional polynomial [15] models were used to describe the dependency of the force of infection on age, as discussed in Shkedy et al. [13]. Briefly, a generalized linear model for the binary data with logit link was used to estimate the force of infection. The linear predictor for that model is given by

$\eta_{m}\left(a, \beta, p_{1}, p_{2}, \ldots, p_{m}\right)=\sum_{i=0}^{m} \beta_{i} H_{i}(a)$,

where $m$ is an integer, $p_{1}<p_{2}<\ldots<p_{m}$ is a sequence of powers and $H_{i}(a)$ is a transformation function given by

$H_{i}(a)= \begin{cases}a^{p_{i}} & \text { if } p_{i} \neq p_{i-1}, \\ H_{i-1}(a) \times \log (a) & \text { if } p_{i}=p_{i-1},\end{cases}$

with $p_{0}=0$ and $H_{0}=1$. As shown in Shkedy et al. [13] the force of infection in this model can be expressed as

$\lambda(a)=\eta_{m}\left(a, \beta, p_{1}, p_{2}, \ldots, p_{m}\right)^{\prime} \frac{\mathrm{e}^{\eta_{m}\left(a, \beta, p_{1}, p_{2}, \ldots, p_{m}\right)}}{1+\mathrm{e}^{\eta_{m}\left(a, \beta, p_{1}, p_{2}, \ldots, p_{m}\right)}}$,

where $\eta_{m}\left(a, \beta, p_{1}, p_{2}, \ldots, p_{m}\right)^{\prime}$ denotes the partial derivative of $\eta_{m}\left(a, \beta, p_{1}, p_{2}, \ldots, p_{m}\right)$ with respect to age $a$. Figure 1 shows, for the varicella dataset, the estimated model for the prevalence of seropositive hosts (Fig. 1a) and the force of infection (Fig. 1b). Constrained fractional polynomials were fitted to ensure that the estimated force of infection will be non-negative. The model fit was based on the value of the Akaike Information Criterion (AIC) and the selected model has exponents $p_{1}=-0.4$ and $p_{2}=-0.3$ with an AIC equal to $125 \cdot 769$.

The estimated force of infection is given by equation (9), with $m=2$ and $\eta_{2}(a)=-40 \cdot 231 a^{-0 \cdot 3}$ $+28 \cdot 153 a^{-0.4}+11 \cdot 303$. According to this model, the force of infection for varicella in Belgium peaks at 2 years of age with a value of $\ell(2)=0.3111$ and drops monotonically for older susceptibles. At 44 years of age the force of infection is estimated to be $0 \cdot 0315$. The mean force of infection for each of the six age groups can be estimated by integrating from the parametric model of the force of infection in equation (9). Hence, we first use a flexible parametric model to estimate the force of infection and integrate over the age groups thereafter. The advantage of using fractional polynomials is that this integration can be performed analytically and the force of infection in age group $i(i=1, \ldots, 6)$ is given by

$\lambda_{i}=\frac{\log \left(\frac{1+\mathrm{e}^{\eta_{2}}\left(a_{i}\right)}{1+\mathrm{e}^{\eta_{2}\left(a_{i-1}\right)}}\right)}{a_{i}-a_{i-1}}$,

where $a_{i-1}$ and $a_{i}$ are the lower and upper bounds of age group $i$, respectively. The estimates for the six age groups were $\hat{\lambda}_{1}=0 \cdot 254, \hat{\lambda}_{2}=0 \cdot 267, \hat{\lambda}_{3}=0 \cdot 160$, 
(a)

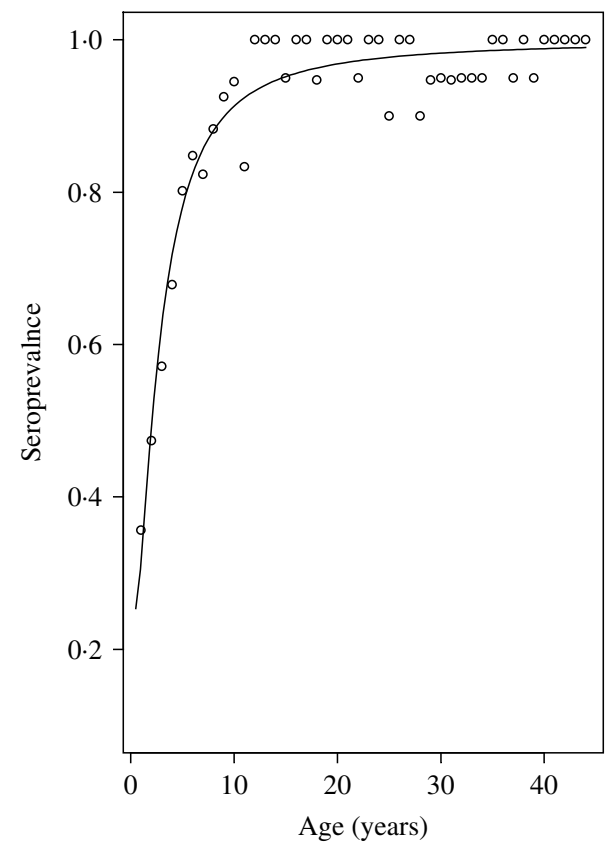

(b)

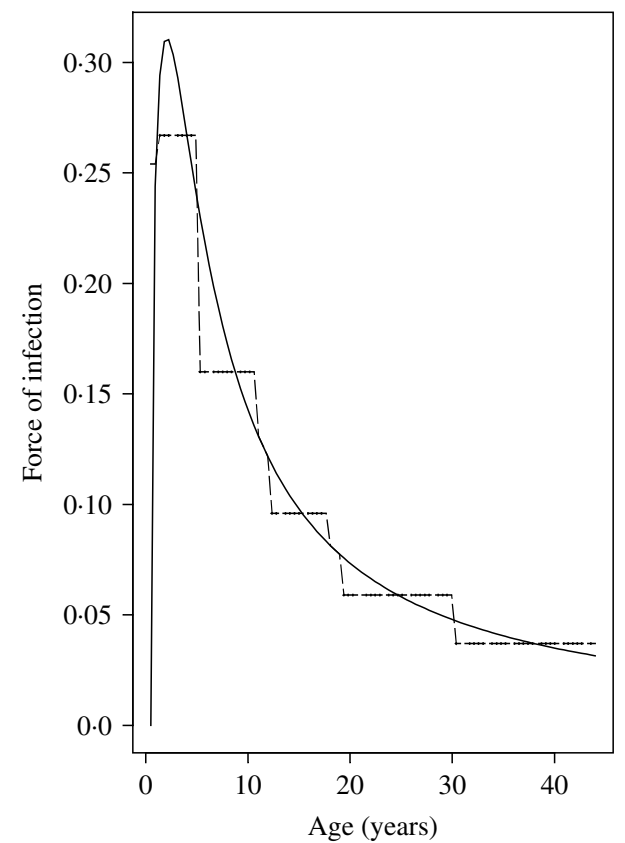

Fig. 1. Estimated prevalence $(a)$ and force of infection $(b)$ for varicella in Belgium. - - - Integrated force of infection.

$\hat{\lambda}_{4}=0 \cdot 096, \hat{\lambda}_{5}=0.059$ and $\hat{\lambda}_{6}=0.037$ for age groups 6 months-1 year, 2-5 years, 6-11 years, 12-18 years, 19-30 years and 31-44 years, respectively. Figure $1 b$ shows the force of infection estimated by the fractional polynomial (solid line) and the integrated force of infection (dashed line) for the six age groups.

\section{Estimation of the transmission coefficients}

Once the estimates of the force of infection are obtained, the elements of the WAIFW matrix can be computed by

$\frac{L}{D \times N} \hat{\lambda}_{i}=\sum_{j=1}^{n} \beta_{i j} \Psi_{j}$

where $L$ is the life-expectancy at birth, $D$ is the mean duration of infectiousness, $N$ is the total population size and $\Psi_{j}$ is given by equation (5). Substituting the $\hat{\lambda}_{i}$ s by their expression, we have

$\mathrm{e}^{-\varphi_{0}}=1$,

$\mathrm{e}^{-\varphi_{1}}=\frac{1+\mathrm{e}^{\eta\left(a_{0}\right)}}{1+\mathrm{e}^{\eta\left(a_{1}\right)}}$

It is easy to show that for $i=2, \ldots, 6$ :

$\mathrm{e}^{-\varphi_{i}}=\Pi_{j=1}^{i} \frac{1+\mathrm{e}^{\eta\left(a_{j-1}\right)}}{1+\mathrm{e}^{\eta\left(a_{j}\right)}}$
Hence, in our model for varicella using six age groups, the expressions for the $\Psi_{i}$ s are

$\Psi_{i}=\mathrm{e}^{-\varphi_{i-1}}-\mathrm{e}^{-\varphi_{i}}=\frac{\left(1+\mathrm{e}^{\eta\left(a_{0}\right)}\right)\left(\mathrm{e}^{\eta\left(a_{i}\right)}-\mathrm{e}^{\eta\left(a_{i-1}\right)}\right)}{\left(1+\mathrm{e}^{\eta\left(a_{i-1}\right)}\right)\left(1+\mathrm{e}^{\eta\left(a_{i}\right)}\right)}$,

for $i=1, \ldots, 6$, where $a_{0}=0 \cdot 5, a_{1}=2, a_{2}=6, a_{3}=12$, $a_{4}=19, a_{5}=31, a_{6}=45, N=10237988, D=7 / 365$ years and $L=78$ years.

System (4) $(i=1, \ldots, n)$ is a linear system of $n$ equations in $n^{2}$ unknowns: the elements of the WAIFW matrix $\beta_{i j}(i, j=1, \ldots, n)$. Since this system is underdetermined, we need to impose a structure upon the WAIFW matrix, limiting the number of unknowns to $n$ ( $n=6$ for our model of varicella). We have estimated the elements of the WAIFW matrix for the six types of matrix structure described above. For example, the estimate of $\beta_{6}$ for the WAIFW matrix structure $W_{V 3}$ is given by

$\beta_{6}=\frac{\frac{L}{D N} \hat{\lambda}_{6}}{\Psi_{1}+\Psi_{2}+\Psi_{3}+\Psi_{4}+\Psi_{5}+\Psi_{6}}$.

The expressions for the other $\beta_{i}$ s are given in the Appendix for matrix $W_{V 3}$. Similar expressions can be derived for each of the five other matrix structures.

The estimates of the $\beta_{i}$ s are given in Table 1 for the six WAIFW matrix structures described above (see also Fig. 2), together with two types of confidence intervals (CIs): the non-parametric bootstrap percentile $95 \%$ 
Table 1. Parameter estimates for the transmission coefficients. Decimal points are shown to illustrate differences in the confidence intervals

\begin{tabular}{|c|c|c|c|c|}
\hline \multirow[b]{2}{*}{ Matrix } & \multirow[b]{2}{*}{ Parameter } & \multirow{2}{*}{$\begin{array}{l}\text { Estimate } \\
\left(\times 10^{-5}\right)\end{array}$} & \multicolumn{2}{|c|}{$95 \%$ confidence intervals $\left(\times 10^{-5}\right)$} \\
\hline & & & Non-parametric & Parametric \\
\hline \multirow[t]{6}{*}{$W_{V 1}$} & $\beta_{1}$ & $28 \cdot 6682$ & $27 \cdot 7358-29 \cdot 5323$ & $27 \cdot 5719-29 \cdot 5306$ \\
\hline & $\beta_{2}$ & $21 \cdot 8524$ & $20 \cdot 3157-23 \cdot 6582$ & $20 \cdot 4073-23 \cdot 7245$ \\
\hline & $\beta_{3}$ & $35 \cdot 1168$ & $29 \cdot 0089-43 \cdot 0572$ & $29 \cdot 8378-43 \cdot 4135$ \\
\hline & $\beta_{4}$ & $54 \cdot 5450$ & $40 \cdot 7348-75 \cdot 4701$ & $42 \cdot 3997-76 \cdot 1505$ \\
\hline & $\beta_{5}$ & $38 \cdot 7205$ & $26 \cdot 5479-58 \cdot 3102$ & $28 \cdot 0602-59 \cdot 4965$ \\
\hline & $\beta_{6}$ & $1 \cdot 5094$ & $1 \cdot 3360-1 \cdot 7058$ & $1 \cdot 3609-1 \cdot 7053$ \\
\hline \multirow{6}{*}{$W_{V 2}$} & $\beta_{1}$ & $11 \cdot 6105$ & $10 \cdot 4412-12 \cdot 7368$ & $10 \cdot 4131-12 \cdot 7302$ \\
\hline & $\beta_{2}$ & $12 \cdot 7365$ & $10 \cdot 8082-14 \cdot 8602$ & $10 \cdot 8940-14 \cdot 6658$ \\
\hline & $\beta_{3}$ & $6 \cdot 7183$ & $5 \cdot 9755-7 \cdot 6053$ & $6 \cdot 0147-7 \cdot 5431$ \\
\hline & $\beta_{4}$ & $3 \cdot 9368$ & $3 \cdot 4821-4 \cdot 4661$ & $3 \cdot 5077-4 \cdot 4259$ \\
\hline & $\beta_{5}$ & $2 \cdot 3970$ & $2 \cdot 1192-2 \cdot 7169$ & $2 \cdot 1350-2 \cdot 6917$ \\
\hline & $\beta_{6}$ & $1 \cdot 5094$ & $1 \cdot 3351-1 \cdot 7081$ & $1 \cdot 3459-1 \cdot 6923$ \\
\hline \multirow{6}{*}{$W_{V 3}$} & $\beta_{1}$ & $10 \cdot 8318$ & $9 \cdot 9369-11 \cdot 7734$ & $9 \cdot 9768-11 \cdot 7272$ \\
\hline & $\beta_{2}$ & $13 \cdot 9985$ & $12 \cdot 5165-15 \cdot 6485$ & $12 \cdot 5210-15 \cdot 5900$ \\
\hline & $\beta_{3}$ & $4 \cdot 5187$ & $2 \cdot 9861-6 \cdot 0658$ & $2 \cdot 9490-6 \cdot 0417$ \\
\hline & $\beta_{4}$ & 3.9368 & $3 \cdot 4693-4 \cdot 4508$ & $3 \cdot 4827-4 \cdot 4379$ \\
\hline & $\beta_{5}$ & $2 \cdot 3970$ & $2 \cdot 1111-2 \cdot 7083$ & $2 \cdot 1205-2 \cdot 6978$ \\
\hline & $\beta_{6}$ & $1 \cdot 5094$ & $1 \cdot 3306-1 \cdot 7022$ & $1 \cdot 3371-1 \cdot 6951$ \\
\hline \multirow[t]{6}{*}{$W_{V 4}$} & $\beta_{1}$ & $10 \cdot 2380$ & $9 \cdot 3540-11 \cdot 2279$ & $9 \cdot 3234-11 \cdot 1187$ \\
\hline & $\beta_{2}$ & $10 \cdot 7494$ & $9 \cdot 6824-12 \cdot 0692$ & $9.6734-11.9518$ \\
\hline & $\beta_{3}$ & $6 \cdot 4435$ & $5 \cdot 6806-7 \cdot 4510$ & $5 \cdot 6677-7 \cdot 2881$ \\
\hline & $\beta_{4}$ & $3 \cdot 8779$ & $3 \cdot 4311-4 \cdot 4542$ & $3 \cdot 4111-4 \cdot 3827$ \\
\hline & $\beta_{5}$ & $2 \cdot 3887$ & $2 \cdot 1112-2 \cdot 7300$ & $2 \cdot 1051-2 \cdot 6929$ \\
\hline & $\beta_{6}$ & $1 \cdot 5094$ & $1 \cdot 3364-1 \cdot 7188$ & $1 \cdot 3334-1 \cdot 6960$ \\
\hline \multirow[t]{6}{*}{$W_{V 5}$} & $\beta_{1}$ & $29 \cdot 8724$ & $28 \cdot 9071-30 \cdot 6015$ & $28 \cdot 9620-30 \cdot 6812$ \\
\hline & $\beta_{2}$ & $22 \cdot 5377$ & $21 \cdot 0784-24 \cdot 2330$ & $21 \cdot 0108-24 \cdot 3728$ \\
\hline & $\beta_{3}$ & $38 \cdot 4309$ & $32 \cdot 1284-46 \cdot 5073$ & $32 \cdot 2774-46 \cdot 5480$ \\
\hline & $\beta_{4}$ & $66 \cdot 7451$ & $50 \cdot 7934-89 \cdot 8739$ & $51 \cdot 2996-90 \cdot 4674$ \\
\hline & $\beta_{5}$ & $62 \cdot 2835$ & $44 \cdot 8055-89 \cdot 4235$ & $45 \cdot 6252-90 \cdot 3289$ \\
\hline & $\beta_{6}$ & 0.9391 & $0 \cdot 7546-1 \cdot 1525$ & $0 \cdot 7657-1 \cdot 1486$ \\
\hline \multirow[t]{6}{*}{$W_{V 6}$} & $\beta_{1}$ & $31 \cdot 8553$ & $31 \cdot 3491-32 \cdot 3493$ & $31 \cdot 3605-32 \cdot 3941$ \\
\hline & $\beta_{2}$ & $23 \cdot 6662$ & $22 \cdot 130-25 \cdot 2923$ & $22 \cdot 0467-25 \cdot 6191$ \\
\hline & $\beta_{3}$ & $43 \cdot 8884$ & $36 \cdot 7338-53 \cdot 0386$ & $36 \cdot 4274-54 \cdot 2846$ \\
\hline & $\beta_{4}$ & $86 \cdot 8345$ & $65 \cdot 136-117 \cdot 256$ & $64 \cdot 743-123 \cdot 908$ \\
\hline & $\beta_{5}$ & $101 \cdot 083$ & $69 \cdot 503-150 \cdot 779$ & $69 \cdot 123-160 \cdot 334$ \\
\hline & $\beta_{6}$ & $162 \cdot 356$ & $103 \cdot 335-263 \cdot 287$ & $102 \cdot 905-280 \cdot 916$ \\
\hline
\end{tabular}

CIs and the parametric bootstrap percentile $95 \%$ CIs. We now provide details on the computations.

\section{Bootstrap confidence intervals}

In the seroprevalence sample, we have 44 samples by age of size $N_{i}(i=1, \ldots, 44)$ and let $p_{i}$ be the proportion of subjects of age $i$ who are seropositive for varicellazoster virus antibodies. The total number of subjects in the seroprevalence sample is $N=\sum N_{i}$. A nonparametric bootstrap sample is a sample of size $N$ obtained by drawing a random sample (with replacement) for each age $i$. The sample for age $i$ is a sample of size $N_{i}$ drawn from a Bernoulli distribution with probability $p_{i}$. Since we are interested in the number of subjects seropositive for varicella-zoster virus in the sample, we can equivalently generate a random value from the binomial distribution $\left(N_{i}, p_{i}\right)$ for each age $i$. We have used 1000 bootstrap samples in the computation since this is deemed necessary for the estimation of confidence intervals. For each bootstrap sample, we can then estimate the parameters of the model. For these estimates, we can estimate the mean force of infection $\lambda_{1}, \lambda_{2}, \ldots, \lambda_{6}$, in each of the six age groups. In this way we obtain 1000 bootstrap values 

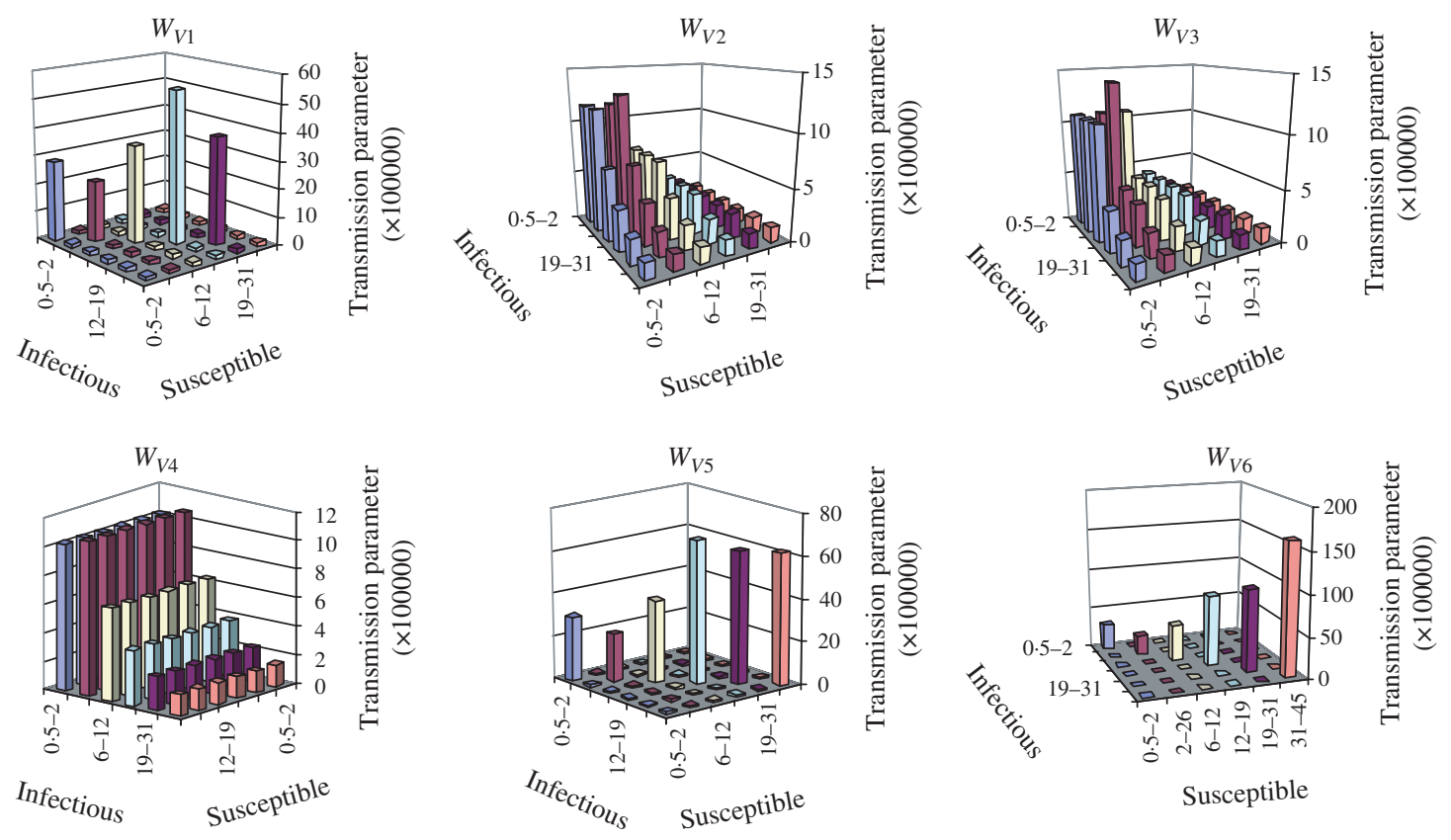

$\square 0.5-2 \mathrm{yr}, \square 2-6 \mathrm{yr}, \square 6-12 \mathrm{yr}, \square 12-19 \mathrm{yr}, \square 19-31 \mathrm{yr}, \square 31-45 \mathrm{yr}$

Fig. 2. WAIFW matrices for varicella in Belgium. Configurations 1-6. The upper limit is not included in the category age.

for each $\lambda_{i}(i=1, \ldots, 6)$. The non-parametric percentile bootstrap $95 \%$ CI for a parameter (for example $\lambda_{1}$ ), is obtained from the bootstrap distribution of $\lambda_{1}$ by taking the 25 th and the 975 th value in the sequentially ordered set of 1000 bootstrap values of this parameter.

The parametric bootstrap and the computation of the parametric percentile bootstrap $95 \%$ CIs is performed in the same way as described above for the non-parametric bootstrap except that instead of drawing for each age $i$ a random sample with replacement from the Bernoulli distribution with probability $p_{i}$, i.e. from the data, we can use instead the parametric model for the cumulative distribution of the age at infection $\pi(a)$ and draw the random sample from the Bernoulli distribution with probability $f_{i}$, where $f_{i}$ is the fitted value for age $i$.

With the matrix structure $W_{V 1}$, the transmission is the highest between hosts aged $12-18$ years $\left(54.5 \times 10^{-5}\right.$, see also Fig. 2). This implies that the highest probability of infection is between an infective aged 12-18 years and a susceptible belonging to the same age group, presumably because of the high frequency of close contacts. In the other age groups, the transmission increases almost monotonically with age for hosts aged $\leqslant 18$ years and decreases monotonically with age for hosts aged $>19$ years. The transmission coefficient among hosts aged 31-44 years, which is also the 'background' transmission coefficient between hosts belonging to different age groups is very low $\left(1.5 \times 10^{-5}\right)$. The two highest transmission coefficients with the matrix structure $W_{V 2}$ are among hosts aged $2-5$ years $\left(12.7 \times 10^{-5}\right)$ and between hosts aged 6 months -1 year and all hosts up to 6 years of age $\left(11 \cdot 6 \times 10^{-5}\right)$. The mixing pattern with matrix $W_{V 3}$ is quite similar to the pattern with $W_{V 2}$. The two highest transmission coefficients with $W_{V 3}$ is among hosts aged $2-5$ years $\left(14 \times 10^{-5}\right)$ and between hosts aged 6 months- 1 year and all hosts up to 12 years of age $\left(10 \cdot 8 \times 10^{-5}\right)$. With matrix structure $W_{V 4}$, the two highest transmission coefficients are for susceptible hosts aged $2-5$ years $\left(10 \cdot 7 \times 10^{-5}\right)$ and hosts aged 6 months -1 year $\left(10 \cdot 2 \times 10^{-5}\right)$. The transmission coefficient decreases monotonically with increasing age for susceptible hosts aged $>2$ years. Below 18 years of age, $W_{V 5}$ and $W_{V 1}$ have similar mixing patterns. Transmission is highest between hosts aged $12-18$ years $\left(66.7 \times 10^{-5}\right)$ and increases almost monotonically with age for hosts aged $\leqslant 18$ years and decreases monotonically with age for hosts aged $>19$ years. However, the transmission coefficient in the 19-30 years age group, which is constrained to be equal to the parameter in the 31-44 years group is much higher than with matrix $W_{V 1}$. Just as with $W_{V 1}$, the 'background' transmission coefficient between hosts belonging to different age groups is very low $\left(0 \cdot 9 \times 10^{-5}\right)$. With matrix $W_{V 6}$ with no transmission at all between hosts belonging to 
different age groups, the transmission coefficient increases almost monotonically with age and is the highest in hosts aged $31-44$ years $\left(162 \cdot 4 \times 10^{-5}\right)$.

\section{Estimation of $\boldsymbol{R}_{0}$ from the WAIFW matrices}

The global basic reproductive number $R_{0}$ for the population is the dominant eigenvalue of the "next generation matrix' whose elements are the individual basic reproductive numbers $R_{0_{i j}}(i=1,2, \ldots, 6, j=1$, $2, \ldots, 6)$ for the transmission of the infection from an infectious person in the age group $j$ to a susceptible person in the age group $i$. By definition of the basic reproductive number, each $R_{0_{i j}}=\beta_{i j} \times N_{i} \times D$, where $\beta_{i j}$ is the $i j$ th element of the WAIFW matrix, $D$ is the mean duration of infectiousness and $N_{i}$ is the total population in age group $i$. For varicella, the mean duration of infectiousness is 7 days $=7 / 365$ years. Like the elements of the WAIFW matrix, the $95 \%$ CIs for $R_{0}$ are computed using two different methods: percentile non-parametric bootstrap and percentile parametric bootstrap.

The minimal immunization coverage needed for elimination, i.e. the proportion of the total population to be immunized immediately after waning of maternal antibodies in order to eliminate varicella, $p_{c}$, is obtained by the relationship:

$p_{c}=1-\frac{1}{R_{0}}$.

\section{Bootstrap confidence interval for estimation of $R_{\mathbf{0}}$ and $p_{c}$}

Table 2 and Figure 3 show the parameter estimates for $R_{0}$ and $p_{c}$. Depending on the configuration of the WAIFW matrix, the basic reproductive number ranges between $3 \cdot 12$ (95\% non-parametric CI 2.78-3.50) for $W_{V 3}$ to 68.57 (95\% non-parametric CI 43.64-111.20) for the assortative mixing pattern $W_{V 6}$. This implies that across the different configurations of the WAIFW matrices $p_{c}$ ranges from $63.99 \%$ (lower limit for $W_{V 3}$ ) to $99 \cdot 10 \%$ (upper limit for the assortative mixing pattern).

\section{DISCUSSION}

When the force of infection is both time and age dependent, the WAIFW matrix is a central parameter in modelling the spread of the infection in the population. A structure has to be assumed for the WAIFW matrix in order to be able to estimate
Table 2. Estimates of the basic reproductive number $R_{0}$ and $p_{c}$ for different matrix structures for the "Who Acquires Infection From Whom' (WAIFW) matrix

\begin{tabular}{|c|c|c|c|c|}
\hline \multirow[b]{2}{*}{ Parameter } & \multirow[b]{2}{*}{ WAIFW } & \multirow[b]{2}{*}{ Estimate } & \multicolumn{2}{|c|}{$95 \%$ confidence intervals } \\
\hline & & & $\begin{array}{l}\text { Non- } \\
\text { parametric }\end{array}$ & Parametric \\
\hline \multirow[t]{6}{*}{$R_{0}$} & $W_{V 1}$ & 11.99 & $8 \cdot 29-17 \cdot 98$ & $8 \cdot 75-18 \cdot 34$ \\
\hline & $W_{V 2}$ & $3 \cdot 19$ & $2 \cdot 87-3 \cdot 57$ & $2 \cdot 89-3 \cdot 54$ \\
\hline & $W_{V 3}$ & $3 \cdot 12$ & $2 \cdot 78-3 \cdot 50$ & $2 \cdot 79-3 \cdot 49$ \\
\hline & $W_{V 4}$ & $4 \cdot 34$ & $3 \cdot 90-4 \cdot 89$ & $3 \cdot 90-4 \cdot 81$ \\
\hline & $W_{V 5}$ & $26 \cdot 33$ & $18 \cdot 95-37 \cdot 79$ & $12 \cdot 29-38 \cdot 17$ \\
\hline & $W_{V 6}$ & $68 \cdot 57$ & $43 \cdot 64-111 \cdot 20$ & $43 \cdot 46-118 \cdot 64$ \\
\hline \multirow{6}{*}{$p_{c}(\%)$} & $W_{V 1}$ & $91 \cdot 66$ & $87 \cdot 94-94 \cdot 44$ & $88 \cdot 57-94 \cdot 55$ \\
\hline & $W_{V 2}$ & $68 \cdot 65$ & $65 \cdot 12-71 \cdot 99$ & $65 \cdot 35-71 \cdot 75$ \\
\hline & $W_{V 3}$ & $67 \cdot 92$ & $63 \cdot 99-71 \cdot 40$ & $64 \cdot 11-71 \cdot 31$ \\
\hline & $W_{V 4}$ & $76 \cdot 95$ & $74 \cdot 39-79 \cdot 55$ & $74 \cdot 38-79 \cdot 19$ \\
\hline & $W_{V 5}$ & $96 \cdot 20$ & $94 \cdot 72-97 \cdot 35$ & $94 \cdot 82-97 \cdot 38$ \\
\hline & $W_{V 6}$ & $98 \cdot 54$ & $97 \cdot 71-99 \cdot 10$ & $97 \cdot 70-99 \cdot 16$ \\
\hline
\end{tabular}

the transmission coefficients and different structures lead to different estimates for the basic reproductive number $R_{0}$ and the minimal immunization coverage needed for elimination of the infection in the population $p_{c}$. In this paper, we have estimated $R_{0}$ and $p_{c}$ for varicella in Belgium for different configurations of the WAIFW matrix. First, the force of infection has been estimated from seroprevalence data stratified by age, using a parametric model with fractional polynomials. The estimates of the mean force of infection over six age groups has then given the means to estimate the transmission coefficients, $R_{\mathbf{0}}$ and $p_{c}$ for six different configurations of the WAIFW matrix. The variability of these parameters has been estimated through the computation of bootstrap confidence intervals. The results show that the values of $R_{0}$ and $p_{c}$ are sensitive to the structure of the WAIFW matrix, with the estimates of $R_{0}$ ranging between 3.12 and 68.57 and those of $p_{c}$ ranging between $67.92 \%$ and $98.54 \%$ for the six configurations chosen. Preliminary empirical data gathered by surveys about the mixing patterns for directly transmitted infections like varicella tend to show that people mostly mix with other people of the same age (configurations $W_{V 1}$, $W_{V 5}$, and $\left.W_{V 6}\right)$. However, although it has the advantage of providing an upper bound on the values of $R_{0}$ and $p_{c}$, configuration $W_{V 6}$ is not realistic since people do not mix exclusively with people of the same age group. Moreover, the assumption of $W_{V 4}$, that only the age of the susceptible hosts matters and not the age of the infectious hosts seems a priori unrealistic. 
(a)

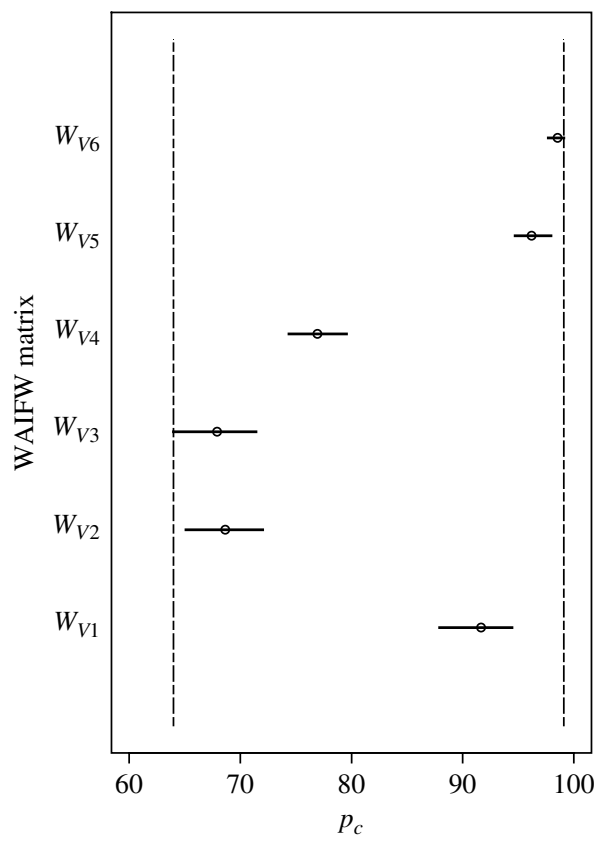

(b)

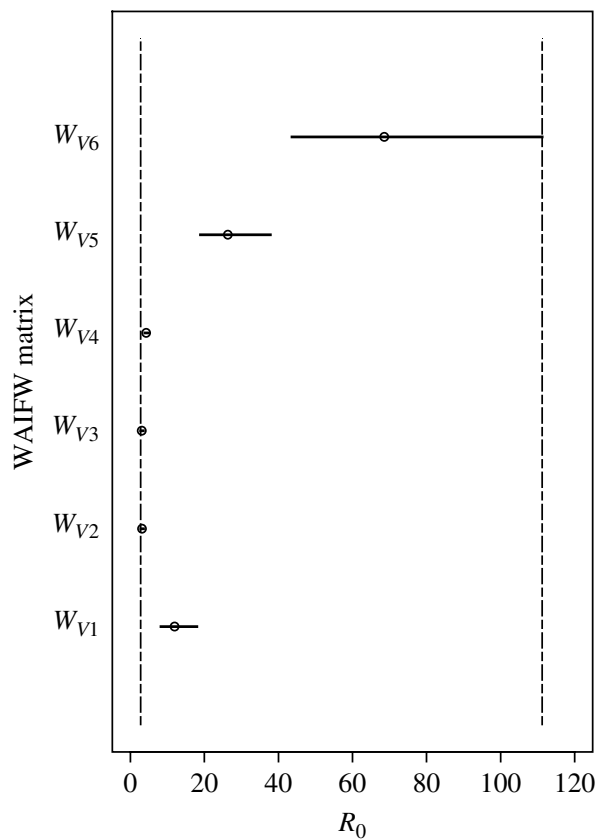

Fig. 3. Estimates for $(a) p_{c}$ and $(b) R_{0}$ and non-parametric bootstrap confidence intervals.

On the other hand, mixing patterns like $W_{V 2}$ and $W_{V 3}$ are probably realistic for a childhood disease like varicella for which it can reasonably be assumed that transmission takes place mainly amongst groups of young children. Hence, configurations $W_{V 1}, W_{V 2}$, $W_{V 3}$, and $W_{V 5}$ are probably the most relevant for varicella, which tends to support a value of $R_{\mathbf{0}}$ between $3 \cdot 12$ and 26.33 and a value of $p_{c}$ between $67.92 \%$ and $96 \cdot 20 \%$. Although using a different model with a force of infection that varies over time and only five age groups, Whitaker \& Farrington [16] obtained similar values for $R_{0}$ for varicella in the United Kingdom. The estimates they obtained with a WAIFW matrix with a configuration similar to our $W_{V 2}$ and $W_{V 3}$ matrices were 3.02 and 3.14 in 1970 and 1998, respectively, while their estimate was 11.79 at both time-points for a WAIFW configuration similar to our assortative $W_{V 1}$ matrix. For elimination to be possible at a critical uptake, $u_{c}$, the vaccine's lifelong efficacy, $e$, should be between $64.0 \%$ and $97 \cdot 4 \%$, respectively, in order for elimination of varicella by vaccination to be theoretically possible (as $u_{c}=p_{c} / e$ ). Different means can be investigated to determine more precisely the value of $R_{0}$ and $p_{c}$. Gathering empirical data about mixing patterns $[17,18]$ should help us to better determine which is the most plausible configuration for a given infection in a population. Another possible avenue is to estimate $R_{\mathbf{0}}$ and $p_{c}$ using seroprevalence data from different infections that have a similar type of transmission, e.g. varicella, parvovirus, measles, mumps and rubella. Assuming a symmetric WAIFW matrix, seroprevalence data from three or four different infections would be enough to estimate the 15 (or 21), transmission coefficients with five (or six), age groups without additional assumptions about the mixing pattern. In any case, it seems clear that further studies on the most appropriate configuration of the WAIFW matrix are necessary to reduce variation in estimated $R_{0}$ and associated parameters.

\section{APPENDIX}

Expression of the $\beta_{i}$ s for the structure $W_{V 3}$ :

$$
\begin{aligned}
& \beta_{6}=\frac{\frac{L}{D N} \hat{\lambda}_{6}}{\Psi_{1}+\Psi_{2}+\Psi_{3}+\Psi_{4}+\Psi_{5}+\Psi_{6}} \\
& \beta_{5}=\frac{\frac{L}{D N} \hat{\lambda}_{5}-\beta_{6} \Psi_{6}}{\Psi_{1}+\Psi_{2}+\Psi_{3}+\Psi_{4}+\Psi_{5}} \\
& \beta_{4}=\frac{\frac{L}{D N} \hat{\lambda}_{4}-\beta_{5} \Psi_{5}-\beta_{6} \Psi_{6}}{\Psi_{1}+\Psi_{2}+\Psi_{3}+\Psi_{4}} \\
& \beta_{1}=\frac{\frac{L}{D N} \hat{\lambda}_{1}-\beta_{4} \Psi_{4}-\beta_{5} \Psi_{5}-\beta_{6} \Psi_{6}}{\Psi_{1}+\Psi_{2}+\Psi_{3}} \\
& \beta_{3}=\frac{\frac{L}{D N} \hat{\lambda}_{3}-\beta_{1} \Psi_{1}-\beta_{4} \Psi_{4}-\beta_{5} \Psi_{5}-\beta_{6} \Psi_{6}}{\Psi_{2}+\Psi_{3}} \\
& \beta_{2}=\frac{\frac{L}{D N} \hat{\lambda}_{2}-\beta_{1} \Psi_{1}-\beta_{3} \Psi_{3}-\beta_{4} \Psi_{4}-\beta_{5} \Psi_{5}-\beta_{6} \Psi_{6}}{\Psi_{2}}
\end{aligned}
$$




\section{ACKNOWLEDGEMENTS}

This paper is based on T.v.E.'s M.Sc. thesis in Biostatistics, at Hasselt University. Z.S., M.A., P.V.D. and P.B. acknowledge funding from 'SIMID', a strategic basic research project of the Institute for the Promotion of Innovation by Science and Technology in Flanders (IWT), project no. 060081 and POLYMOD, a European Commission project funded within the Sixth Framework Programme, Contract no.: SSP22-CT-2004-502084. Z.S., M.A. and G.M. gratefully acknowledge the financial support from the IAP research network no. P5/24 of the Belgian Government (Belgian Science Policy).

\section{DECLARATION OF INTEREST}

This work is unrelated to T.v.E.'s work at GlaxoSmithKline Biologicals where he is employed as a mathematical modeller.

\section{REFERENCES}

1. Anderson RM, May RM. Infectious Diseases of Humans, Dynamic and Control. Oxford University Press, 1991.

2. Halloran ME, Watelet L, Struchiner CJ. Epidemiologic effects of vaccinations with complex direct effects in age-structured population. Mathematical Biosciences 1994; 121: 193-225.

3. Anderson RM, May RM. Age-related changes in the rate of disease transmission: implication for the design of vaccination programmes. Journal of Hygiene (Cambridge) 1985; 94: 365-436.

4. Farrington CP, Kanaan MN, Gay NJ. Estimation of the basic reproduction number for infectious diseases from age-stratified serological survey data (with Discussion). Journal of the Royal Statistical Society, Series C: Applied Statistics 2001; 50: 251-292.

5. Greenhalgh D, Dietz K. Some bounds on estimation for reproductive ratios derived from the age-specific force of infection. Mathematical Biosciences 1994; 124: 9-57.

6. Wallinga $\mathbf{J}$, et al. Estimation for measles reproduction ratios and prospects for elimination of measles by vaccination in some western european countries. Epidemiology and Infection 2001; 127: 281-295.

7. Greenhalgh, D. Discussion in Farrington et al. (2001), Estimation of the basic reproduction number for infectious diseases from age-stratified serological survey data. Journal of the Royal Statistical Society, Series C: Applied Statistics 2001; 50: 251-292.

8. Keiding N. Age-specific incidence and prevalence: a statistical perspective. Journal of the Royal Statistical Society, Series A 1991; 154: 371-412.

9. Shkedy Z, et al. Modeling hepatitis A force of infection using monotone local polynomials. Journal of the Royal Statistical Society, Series C: Applied Satistics 2003; 52: 469-485.

10. Grenfell BT, Anderson RM. The estimation of agerelated rates of infection from case notification and serological data. Journal of Hygiene (Cambridge) 1985; 95: 419-436.

11. Farrington CP. Modeling forces of infection for measles, mumps and rubella. Statistics in Medicine 1990; 9: 953-967.

12. Keiding $\mathbf{N}$, et al. Estimation from current status data in continuous time. Lifetime Data Analysis 1996; 2: 119-129.

13. Shkedy Z, et al. Modeling age dependent force of infection from prevalence data using fractional polynomials. Statistics in Medicine 2006; 25: 15771591.

14. Thiry $\mathbf{N}$, et al. The seroepidemiology of primary varicella-zoster virus infection in Flanders (Belgium). European Journal of Pediatrics 2002; 161: 588-593.

15. Royston P, Altman DG. Regression using fractional polynomials of continuous covariates: parsimonious parametric modeling. Applied Statistics 1994; 43: 429-467.

16. Whitaker HJ, Farrington CP. Infections with varying contact rates: application to varicella. Biometrics 2004; 60: 615-623.

17. Edmunds WJ, O'Callahgan CJ, Nokes DJ. Who mixes with whom? A method to determine the contact patterns of adults that may lead to the spread of airborne infections. Proceedings of the Royal Society of London, Series B 1997; 264: 949-957.

18. Beutels $\mathbf{P}$, et al. Social mixing patterns for transmission models of close contact infections: exploring selfevaluation and diary-based data collection through a web-based interface. Epidemiology and Infection 2006; 134: 1158-1166. 